\title{
Percutaneous mechanical thrombectomy in patients with high-risk pulmonary embolism and contraindications for thrombolytic therapy
}

\author{
Matjaz Bunc ${ }^{1,2}$, Klemen Steblovnik ${ }^{1}$, Simona Zorman ${ }^{3}$, Peter Popovic ${ }^{4}$ \\ ${ }^{1}$ Clinical Department for Cardiology, University Medical Centre Ljubljana, Ljubljana, Slovenia \\ ${ }^{2}$ Institute for Pathophysiology, School of Medicine Ljubljana, Ljubljana, Slovenia \\ ${ }^{3}$ Clinical Department for Intensive Care, University Medical Centre Ljubljana, Ljubljana, Slovenia \\ ${ }^{4}$ Institute of Radiology, University Medical Centre Ljubljana, Ljubljana, Slovenia
}

Radiol Oncol 2020; 54(1): 62-67.

Received 24 November 2019

Accepted 23 January 2020

Correspondence to: Assoc. Prof. Peter Popovic, M.D., Ph.D., University Medical Centre Ljubljana, Clinical Institute of Radiology, Zaloska cesta 7, SI-1525 Ljubljana, Slovenia. E-mail: peter.popovic@kclj.si

Disclosure: No potential conflicts of ineterst were disclosed.

\begin{abstract}
Background. High-risk pulmonary embolism is associated with a high early mortality rate. We report our experience with percutaneous mechanical thrombectomy in patients with high-risk pulmonary embolism and contraindications for thrombolytic therapy.

Patients and methods. This was a retrospective analysis of consecutive patients with high-risk pulmonary embolism and contraindications to thrombolytic therapy. They were treated with percutaneous mechanical thrombectomy which included thrombectomy and additional thrombus aspiration when needed. Clinical parameters and survival to discharge were measured.

Results. From November 2005 to September 2015 we treated 25 patients with a mean age of $62.6 \pm 12.7$ years, $64 \%$ were men. Mean simplified Pulmonary Embolism Severity Index was 2.9. Mean maximum lactate levels were $7.8 \pm 6.6$ $\mathrm{mmol} / \mathrm{L}$, vasopressors were used in $77 \%$, and $59 \%$ needed mechanical ventilation. Mechanical treatment included thrombus fragmentation complemented with aspiration (56\%) and aspiration using Aspirex®S catheter (44\%). Local (5 patients; $20 \%$ ) and systemic (3 patients; $12 \%$ ) thrombolytics were used as a salvage therapy. We observed nonsignificant improvements in systemic blood pressure (100 $\pm 41 \mathrm{~mm} \mathrm{Hg}$ vs $119 \pm 34 ; \mathrm{p}=0.100)$ and heart frequency $(99 \pm 35$ $\mathrm{min}^{-1}$ vs $87 \pm 31 \mathrm{~min}^{-1} ; \mathrm{p}=0.326$ ) before and after treatment, respectively. Peak systolic tricuspid pressure gradient was significantly lower after treatment ( $57 \pm 14 \mathrm{~mm} \mathrm{Hg}$ vs $31 \pm 3 \mathrm{~mm} \mathrm{Hg} ; \mathrm{p}=0.018)$. Overall the procedure was technically successful in 20 patients (80\%) and 17 patients (68\%) survived to hospital discharge.
\end{abstract}

Conclusions. In patients with high-risk pulmonary embolism who cannot receive thrombolytic therapy, percutaneous mechanical thrombectomy is a promising alternative to reduce pulmonary artery pressure.

Key words: high-risk pulmonary embolism; treatment; percutaneous mechanical thrombectomy

\section{Introduction}

Pulmonary embolism (PE) remains a significant cause of cardiovascular morbidity and mortality worldwide, with overall in-hospital mortality rates ranging from $25 \%$ for patients with cardiogenic shock to $65 \%$ for those requiring cardiopulmonary resuscitation. ${ }^{1,2}$
Current guidelines suggest classification of pulmonary embolism severity and the risk of early (in-hospital or 30 day) death into high-, intermediate- and low-risk. ${ }^{3}$ Patients with PE presenting with shock or hypotension are at high risk of in-hospital death; high-risk pulmonary embolism (HRPE). ${ }^{3}$ Most deaths occur within the first few days after diagnosis mostly due to acute right ventricular failure. . $^{3,4}$ 
Treatment of HRPE is based on hemodynamic and respiratory support, unfractionated heparin infusion, and reperfusion therapy with systemic thrombolytic agents (class IB), surgical pulmonary embolectomy (class IC) or percutaneous catheterdirected (mechanical) thrombectomy (PMT) (class $\mathrm{IIaC}){ }^{3}$ Experimental evidence also suggests that extracorporeal cardiopulmonary support can be an effective treatment especially as a bridge to surgical pulmonary embolectomy. ${ }^{5-7}$ PMT improves pulmonary flow with embolus/thrombus modification and may be particularly useful if contraindications to fibrinolysis are present and surgical embolectomy is not feasible or available. PMT has been shown effective in patients with HRPE. ${ }^{3}$ However, no large or solid data regarding the efficacy and safety of PMT treatment of pulmonary embolism are available.

The objective of this retrospective study is to evaluate the immediate haemodynamic effects of PMT in patients with HRPE and contraindications for thrombolytic therapy. Our secondary objective was to compare technical sucess and in-hospital mortality in patients who did and did not receive adjunctive thrombolytic therapy.

\section{Patients and methods}

\section{Patients}

A retrospective review was performed of 25 consecutive patients with high-risk pulmonary embolism over a 10-year period (from November 2005 to September 2015) who had been referred to our department for pulmonary digital subtraction angiography (DSA) and potential catheter intervention. The protocol was approved by the Slovenian National Ethics Committee (Number 0120-124/2018/4) which waived the need for informed consent. The criteria for study inclusion were patients with HRPE and contraindications to thrombolytic therapy. The clinical definition of HRPE was established in the presence of cardiogenic shock or hypotension, the latter defined as systemic systolic blood pressure (sSBP) $<90 \mathrm{~mm}$ $\mathrm{Hg}$, or a pressure drop $\geq 40 \mathrm{~mm} \mathrm{Hg}$ for $>15 \mathrm{~min}$ not caused by arrhythmia, hypovolemia, or sepsis. The diagnosis was made by computed tomography angiography in 24 of 25 cases. In addition, transthoracic echocardiography was performed in 11 patients. The study included 25 patients $(62.6 \pm 12.7$ years; 16 men, 9 women). The youngest patient was 32 and the oldest 81 years old. The most common presenting symptom was dyspnoea $(\mathrm{n}=14 ; 56 \%)$ followed by syncope $(\mathrm{n}=8 ; 32 \%)$, chest pain $(\mathrm{n}=7$; $28 \%)$, cardiac arrest $(4 ; 16 \%)$ and cough $(n=2 ; 8 \%)$. All patients had contraindications for thrombolytic therapy, including 12 with recent major surgery, 4 with ongoing or recent bleeding, 3 with neoplastic disease, 3 with traumatic injuries, two with suspected bleeding and one with recurrent pulmonary embolisms despite thrombolytic therapy. Despite contraindications a salvage thrombolytic therapy was used in 8 of $25(32 \%)$ patients. $3(12 \%)$ patients received systemic fibrinolytic agents during resuscitation in addition to PMT. In the rest 5 (20\%) patients local thrombolysis was introduced after PMT according to operator preferences as on top salvage therapy.

\section{Procedures}

Our standard protocol for percutaneous mechanical thrombectomy includes the use of a long sheath for both percutaneous embolectomy with thrombectomy devices and thrombus fragmentation complemented with manual thrombus aspiration with an aspiration catheter. After local anaesthesia, a 6 French (F) short introducer sheath (Cordis Corp., Miami, FL, USA) is placed in the right or left common femoral vein and access to the pulmonary arteries is obtained with a $5 \mathrm{~F}$ pigtail catheter (Cordis Corp., Miami, FL, USA) advanced over 0.035-inch guide wires (Cordis Corp., Miami, FL, USA). Through the pigtail catheter in the pulmonary artery, a $260 \mathrm{~cm}$ guidewire (Amplatz Super Stiff, Boston Scientific Corporation, Natick, MA) is placed in the peripheral pulmonary artery. The $6 \mathrm{~F}$ sheath is exchanged over the guidewire for an adapted $90 \mathrm{~cm}$ long $8 \mathrm{~F}$ or $12 \mathrm{~F}$ introducer sheath (Cook, Bloomington, USA). The introducer sheath is placed in either the right or the left main pulmonary artery. The 0.035 -inch $180 \mathrm{~cm}$ angled hydrophilic guidewire (Terumo Glidewire, Somerset, USA) is passed through the thrombus and left in a peripheral part of the pulmonary artery. With the guidewire remaining in the peripheral pulmonary artery, the $5 \mathrm{~F}$ or $6 \mathrm{~F}$ pigtail catheter is inserted. The $6 \mathrm{~F}$ catheter with a $10 \mathrm{~mm}$ pigtail wrap is used in the central portion of the pulmonary artery, whereas the $5 \mathrm{~F}$ catheter with a $6 \mathrm{~mm}$ pigtail wrap is used in the peripheral part of the pulmonary artery. The distal end of the pigtail catheter is placed distaly to the thrombus, then the catheter is spun quickly so that the distal curve serves as a rotor blade to fragment the thrombus. The catheter is rotated manually around the axis of the stationary guidewire. After pigtail thrombus fragmentation, 
TABLE 1. Effects of mechanical percutaneous mechanical thrombectomy (PMT)

\begin{tabular}{|c|c|c|c|}
\hline Clinical characteristic $(n=25)$ & Before PMT & After PMT & $P$ value \\
\hline Heart rate, min $^{-1}$ & $100.2 \pm 34.5$ & $86.6 \pm 31.3$ & 0.326 \\
\hline Arterial systolic pressure, $\mathrm{mm} \mathrm{Hg}$ & $91.6 \pm 40.0$ & $121.9 \pm 34.3$ & 0.100 \\
\hline $\begin{array}{l}\text { Peak systolic tricuspid pressure } \\
\text { gradient, } \mathrm{mm} \mathrm{Hg}\end{array}$ & $55.4 \pm 13.3$ & $29.8 \pm 5.2$ & 0.018 \\
\hline
\end{tabular}

Mean \pm standard deviation is shown.

we sometimes performed additional manual clot aspiration with large-lumen percutaneous transluminal coronary angioplasty guide catheter (8-Fr Guider-Softip; Boston Scientific; Scimed or Brite tip; Cordis; Johnson and Johnson, Florida, USA). Strong manual aspiration is created through a regular Luer-lock $50 \mathrm{ml}$ syringe plunger while slowly withdrawing the catheter through the introducer long sheath. To evaluate the distal embolization resulting from catheter thrombectomy, pulmonary angiography is performed several times during these procedures (flow $5 \mathrm{ml} / \mathrm{sec} ; 10 \mathrm{ml}$ of contrast media). In cases, without thrombus fragmentation, we used Aspirex®S $8 \mathrm{~F}$ and $11 \mathrm{~F}$ catheter device (Straub Medical AG, Wangs, Switzerland). Aspirex®S is introduced through a $12 \mathrm{~F}$ introducer sheath (Cook, Bloomington, USA) over an exchange guide wire and then advanced to the occlusion site. The catheter is gently withdrawn and pulled back during aspiration. The heart rate and blood pressure were monitored during the whole procedures. Thrombectomy was discontinued as soon as systemic arterial pressure increased. Technical success was defined as an angiographic improvement of pulmonary flow. At the beginning of each procedure, all patients received 5,000 international units of unfractionated heparin intravenously. After the procedure patients were treated with UHF, guided by aPTT until haemodynamic and respiratory stabilisation and until kidney function was stable. After that LMWH or oral anticoagulant was started. Thrombolytic threatment was provided using alteplase as systemic or local therapy. Systemic therapy included a total of 100 $\mathrm{mg}$ of alteplase as a 10-50 mg slow bolus (1-2 min) and the rest as 2 hour intravenous infusion. Local therapy was individualized and was administered as alteplase infused over pulmonary artery sheath at the rate of $1 \mathrm{mg} / \mathrm{h}$ for up to 15 hours.

\section{Follow-up}

The clinical status, systemic blood pressures, bleeding, and transthoracic echocardiography of all patients were recorded after the procedure and before they were discharged. All patients received warfarin for at least 6 months. Survival to hospital discharge was monitored. Significant bleeding was defined as BARC 3 and $5 .{ }^{8}$

\section{Statistical analysis}

All quantitative data were expressed as mean \pm standard deviation (SD). Dichotomous variables are expressed as percentages. $\mathrm{p}<0.05$ was considered significant. Means were compared using the Paired-Samples T-Test.

\section{Results}

25 patients met the inclusion criteria for the study. The majority of patients were in obstructive shock at presentation with mean maximum lactate levels of 7,8 $\pm 6,6 \mathrm{mmol} / \mathrm{L}$, vasopressors were used in $77 \%$ and $59 \%$ needed mechanical ventilation, veno-arterial extracorporeal membrane oxygenation was used in one case. Mean simplified Pulmonary Embolism Severity Index (sPESI) was 2.9. Fourteen (56\%) patients underwent thrombus fragmentation using a pigtail catheter which was complemented with manual thrombus aspiration in 7 patients. The Aspirex®S percutaneous thrombectomy device was used in $11(44 \%)$ patients. In one patient, treated with Aspirex ${ }^{\circledR}$, additional manual aspiration of the peripheral thrombosis was performed for even better pulmonary perfusion. In one patient surgical embolectomy with right atrium thrombus removal was performed three days before PMT. Seventeen patients (68\%) were treated with PMT only, $3(12 \%)$ patients received systemic fibrinolytic agents during resuscitation in addition to PMT and in $5(20 \%)$ patients local thrombolysis was introduced after PMT according to operator preferences as on top therapy.

We observed nonsignificant improvements in arterial systolic blood pressure $(92 \pm 40 \mathrm{~mm} \mathrm{Hg}$ vs $119 \pm 34 ; \mathrm{p}=0.100)$ and heart rate $\left(99 \pm 35 \mathrm{~min}^{-1}\right.$ vs $87 \pm 31 \mathrm{~min}^{-1} ; \mathrm{p}=0.326$ ) before and after treatment, respectively. Peak systolic tricuspid pressure gradient was significantly lower after treatment (57 \pm $14 \mathrm{~mm} \mathrm{Hg}$ to $31 \pm 3 \mathrm{~mm} \mathrm{Hg}$; p = 0.018) (Table 1). There was no diference in arterial systolic blood pressure, heart rate or peak systolic tricuspid pressure gradient pre and post procedure between patients treated with PMT only and those with additional thrombolysis. 
TABLE 2. Characteristics of the study group

\begin{tabular}{|c|c|c|c|c|}
\hline Clinical characteristic & $\begin{array}{l}\text { All patients } \\
(\mathrm{n}=25)\end{array}$ & $\begin{array}{l}\text { PMT without lysis } \\
\quad(n=17)\end{array}$ & $\begin{array}{l}\text { Systemic or local lysis } \\
+ \text { PMT }(n=8)\end{array}$ & p-value \\
\hline Age, years & $62.6 \pm 12.7$ & $66.1 \pm 11.2$ & $55.4 \pm 13.5$ & 0.05 \\
\hline Breathing frequency before, min $^{-1}$ & $21.6 \pm 10.1$ & $24.4 \pm 8.6$ & $16.8 \pm 12.0$ & 0.25 \\
\hline sPESI score & $2.9 \pm 0.7$ & $3.1 \pm 0.7$ & $2.6 \pm 0.5$ & 0.11 \\
\hline Max. lactate, $\mathrm{mmol} / \mathrm{L}$ & $7.8 \pm 6.6$ & $5.5 \pm 7.1$ & $10.7 \pm 4.6$ & 0.10 \\
\hline Max troponin I, $\mu \mathrm{g} / \mathrm{L}$ & $5.6 \pm 10.8$ & $2.4 \pm 3.4$ & $10.6 \pm 16.3$ & 0.09 \\
\hline Technical success, $\%$ & 80 & 82 & 75 & 0.67 \\
\hline Survival to hospital discharge, $\%$ & 68 & 76 & 50 & 0.19 \\
\hline Cardiogenic schock, \% & 67 & 54 & 88 & 0.11 \\
\hline Mechanical ventilation, \% & 61 & 47 & 88 & 0.06 \\
\hline Vasopressors or inotropes, \% & 77 & 71 & 88 & 0.18 \\
\hline Bleeding, \% & 16 & 12 & 25 & 0.40 \\
\hline Transfusion, \% & 24 & 12 & 50 & 0.04 \\
\hline Aspirex, \% & 44 & 53 & 25 & 0.19 \\
\hline
\end{tabular}

Mean \pm standard deviation is shown if not stated otherwise; NS = not significant $(p>0.05)$; PMT = percutaneous mechanical thrombectomy; sPESI = Simplified Pulmonary Embolism Severity Index

Patients treated with a combination of PMT and thrombolysis were younger $(55.4 \pm 13.5$ vs $66.1 \pm$ 11.2 years respectively; $p=0.05$ ) than patients treated with PMT alone. There was, however, no difference in maximum lactate, troponin I level, sPESI score, mechanical ventilation, cardiogenic schock, vasopressors/inotrope use, bleeding, transfusion or Aspirex use between patients of the two groups (Table 2). There were more transfusions in patients with thrombolysis $(12 \%$ vs $50 \%$; $\mathrm{p}=0.04)$.

Overall PMT was technically successful in 20 patients $(80 \%)$ and 17 patients (68\%) survived to hospital discharge. Technical success and survival to hospital discharge were not significantly different between patients with PMT only and those with PMT plus thrombolysis $(82 \%$ vs $75 \%$ and $76 \%$ vs $50 \%$, respectively) (Table 2 ).

Aside from one BARC $3 \mathrm{~b}$ puncture site bleeding there were no major procedural complications. Minor complications developed in 6 of 25 patients (24\%): transient bradycardia during the catheterization in five patients and groin hematoma in one patient. An inferior vena cava filter (Bard, Crawley, $\mathrm{UK})$ was inserted in nine patients ( $36 \%$ ).

\section{Discussion}

Our single center retrospective study demonstrated the efficacy and safety of PMT in patients with HRPE. HRPE remains an important clinical prob- lem with a high mortality rate. Systemic thrombolytic therapy is currently indicated on top of heparin anticoagulation for acute HRPE accompanied by hemodynamic instability. ${ }^{3}$ But systemic thrombolysis carries a significant risk of bleeding which is approximately $13 \%$ of major bleeding and $1.8 \%$ of intracranial or fatal haemorrhage, particularly, when pre-disposing conditions or comorbidities existed. ${ }^{3}$

If absolute contraindications to thrombolysis are present and if performed in an experienced center in HRPE surgical embolectomy, in addition to anticoagulation, is often used., ${ }^{3,9-11}$ Preoperative thrombolysis increases the risk of bleeding, but it is not an absolute contraindication to surgical embolectomy. ${ }^{12}$ In the case of early embolectomy before the hemodynamic collapse, perioperative mortality rates of $6 \%$ or less have been reported. ${ }^{13,14}$ Pulmonary embolectomy is technically a relatively simple operation, but only a few tertiary care centers offer emergency surgical embolectomy with round-the-clock availability. Additionally in the sickest of patients in severe shock or cardiac arrest time needed to perform surgery may change the prognosis of the patient. In these unstable patients with HRPE PMT may be an effective and safe treatment for improvement of pulmonary flow with thrombus modification. ${ }^{15}$

The goal of interventional treatment is the improvement of pulmonary flow with the removal or distal embolization of obstructing thrombi from 
the main pulmonary arteries to facilitate right ventricle recovery. Acute increase of pulmonaty artery pressure leeds to right ventricle failure which is a predictor of mortality. ${ }^{16,17}$ We showed that PMT can reduce pulmonary artery pressure. In patients threatened by right ventricular failure, even a small hemodynamic improvement may be life-saving and extend the critical time frame for further recanalization. Moreover, the increased total surface area of the fragments may accelerate the efficacy of spontaneous intrinsic lytic activity. ${ }^{18}$

Although we only included patients with contraindications for systemic thrombolysis some of the patients received local thrombolysis and some even systemic thrombolysis as a salvage therapy. Not many clinical data is available about PMT. In a review on interventional treatment that included 35 non-randomized studies $67 \%$ of patients received additional thrombolysis. ${ }^{3}$ Our data proved the effectiveness of PMT also in combination with salvage thrombolytic therapy. The effectiveness of the PMT alone was similar to combined therapy of PMT and thrombolysis. Conversely, if further pharmacologic thrombolysis is desired following primary PMT, an extended thrombolytic infusion can still be performed. ${ }^{19,20}$ Even more, after fragmentation, a greater surface area of thrombus can be exposed to the thrombolytic; consequently, less drug and perhaps less time is needed to achieve thrombolysis. However, although we failed to show any difference hospital survival in patients with or without thrombolytic therapy, there is a trend to increased mortality in patients who received thrombolysis. This is probably due to worse clinical condition of patients who received thrombolysis (trends towards higher maximal lactate and troponin, more mechanical ventilation, vasopressor and inotrope use and more cardiogenic schock). Interestingly the sPESI score does not show the difference between the groups which could reflect the fact that it was calculated at addmision and patients condition often deteriorated afterwards. We also failed to show a difference in bleeding between patients with or without thrombolytic therapy. This is probably due to small sample size. However, puncture of a femoral vein (even with $12 \mathrm{~F}$ sheath) does not seam to bring a high additional risk since we only had one procedure related bleeding event. Although there was no difference in bleeding we observed more transfusions in patients with thrombolysis. This was due to non-bleeding related transfusions (anemia or suspected bleeding).
Veno-arterial extracorporeal membrane oxygenation could be used to rescue patients when thrombolytic treatment fails or as temporary hemodynamic support prior to surgical ${ }^{21}$ or catheter-based embolectomy. ${ }^{22}$

Our study has limitations. First, it is a retrospective, single-center study. Second, patients were treated according to the available strategy, not systematic indications. This includes individualized thrombolytic treatment. Also due to a very short timeframe available in these critically ill patients not all the patients had echocardiography done before the PMT and thus peak systolic tricuspid pressure gradient difference is of limited value. Due to retrospective nature of the study and a long period of inclusion some data are missing. Third, we did not perform a protocol-based follow-up based on long-term echocardiography and imaging to detect chronic thromboembolic pulmonary hypertension development.

In conclusion, in patients with high-risk pulmonary embolism who cannot receive thrombolytic therapy, percutaneous mechanical thrombectomy is a promising alternative to reduce pulmonary artery pressure.

\section{References}

1. Agnelli G, Becattini C. Acute pulmonary embolism. N Engl J Med 2010; 363: 266-74. doi: 10.1056/NEJMra0907731

2. Kasper W, Konstantinides S, Geibel A, Olschewski M, Heinrich F, Grosser KD, et al. Management strategies and determinants of outcome in acute major pulmonary embolism: results of a multicenter registry. J Am Coll Cardiol 1997; 30: 1165-71. doi: 10.1016/s0735-1097(97)00319-7

3. Konstantinides SV, Meyer G, Becattini C, Bueno H, Geersing GJ, Harjola VP, et al. 2019 ESC Guidelines for the diagnosis and management of acute pulmonary embolism developed in collaboration with the European Respiratory Society (ERS). Eur Heart J 2020; 41: 543-603. doi: 10.1093/eurheartj/ehz405

4. Kucher N, Rossi E, De Rosa M, Goldhaber SZ. Massive pulmonary embolism. Circulation 2006; 113: 577-82. doi: 10.1161/CIRCULATIONAHA.105.592592

5. Kjærgaard B, Rasmussen BS, de Neergaard S, Rasmussen LH, Kristensen SR. Extracorporeal cardiopulmonary support may be an efficient rescue of patients after massive pulmonary embolism. An experimental porcine study. Thromb Res 2012; 129: e147-51. doi: 10.1016/j.thromres.2012.01.007

6. Delnoij TSR, Accord RE, Weerwind PW, Donker DW. Atrial trans-septal thrombus in massive pulmonary embolism salvaged by prolonged extracorporeal life support after thrombo-embolectomy. A bridge to rightsided cardiovascular adaptation. Acute Card Care 2012; 14: 138-40. doi: 10.3109/17482941.2012.741247

7. Leick J, Liebetrau C, Szardien S, Willmer M, Rixe J, Nef H, et al. Percutaneous circulatory support in a patient with cardiac arrest due to acute pulmonary embolism. Clin Res Cardiol 2012; 101: 1017-20. doi: 10.1007/s00392-012 0481-x

8. Mehran R, Rao S V, Bhatt DL, Gibson CM, Caixeta A, Eikelboom J, et al Standardized bleeding definitions for cardiovascular clinical trials: a consensus report from the Bleeding Academic Research Consortium. Circulation 2011;123: 2736-47. doi: 10.1161/CIRCULATIONAHA.110.009449

9. Goldhaber SZ. Percutaneous mechanical thrombectomy for acute pulmonary embolism: a double-edged sword. Chest 2007; 132: 363-5. doi: 10.1378/chest.07-0591 
10. Kucher N, Goldhaber SZ. Management of massive pulmonary embolism. Circulation 2005; 112: e28-32. doi: 10.1161/CIRCULATIONAHA.105.551374

11. Shiomi D, Kiyama H, Shimizu M, Yamada M, Shimada N, Takahashi A, et al. Surgical embolectomy for high-risk acute pulmonary embolism is standard therapy. Interact Cardiovasc Thorac Surg 2017; 25: 297-301. doi: 10.1093/ icvts/ivx091

12. Aklog L, Williams CS, Byrne JG, Goldhaber SZ. Acute pulmonary embolectomy: a contemporary approach. Circulation 2002; 105: 1416-9. doi 10.1161/01.cir.0000012526.21603.25

13. Leacche M, Unic D, Goldhaber SZ, Rawn JD, Aranki SF, Couper GS, et al. Modern surgical treatment of massive pulmonary embolism: results in 47 consecutive patients after rapid diagnosis and aggressive surgical approach. J Thorac Cardiovasc Surg 2005; 129: 1018-23. doi: 10.1016/j. jtcvs.2004.10.023

14. Malekan R, Saunders PC, Yu CJ, Brown KA, Gass AL, Spielvogel D, et al. Peripheral extracorporeal membrane oxygenation: comprehensive therapy for high-risk massive pulmonary embolism. Ann Thorac Surg 2012; 94: 104 8. doi: 10.1016/j.athoracsur.2012.03.052

15. Spies C, Khandelwal A, Smith TH, Jolly N, Kavinsky CJ. Percutaneous mechanical thrombectomy for massive pulmonary embolism using conservative treatment strategy. J Interv Cardiol 2008; 21: 566-71. doi: 10.1111/j.1540-8183.2008.00405.x

16. Quiroz R, Kucher N, Schoepf UJ, Kipfmueller F, Solomon SD, Costello P, et al. Right ventricular enlargement on chest computed tomography: prognostic role in acute pulmonary embolism. Circulation 2004; 109: 2401-4. doi: 10.1161/01.CIR.0000129302.90476.BC

17. Dahhan T, Siddiqui I, Tapson VF, Velazquez EJ, Sun S, Davenport CA, et al. Clinical and echocardiographic predictors of mortality in acute pulmonary embolism. Cardiovasc Ultrasound 2016; 14: 44. doi: 10.1186/s12947-0160087-y

18. Zhou WZ, Shi HB, Yang ZQ, Liu S, Zhou CG, Zhao LB, et al. Value of percutanous catheter fragmentation in the management of massive pulmonary embolism. Chin Med J (Engl) 2009; 122: 1723-7. doi: 10.3760/cma.j.is sn.0366-6999.2009.15.001

19. Kuo WT, Gould MK, Louie JD, Rosenberg JK, Sze DY, Hofmann L V. Catheterdirected therapy for the treatment of massive pulmonary embolism: systematic review and meta-analysis of modern techniques. J Vasc Interv Radiol 2009; 20: 1431-40. doi: 10.1016/j.jvir.2009.08.002

20. Sag S, Nas OF, Kaderli AA, Ozdemir B, Baran I, Erdoğan C, et al. Catheter directed ultrasound-accelerated thrombolysis may be life-saving in patients with massive pulmonary embolism after failed systemic thrombolysis. J Thromb Thrombolysis 2016; 42: 322-8. doi: 10.1007/s11239-016-1370-3

21. Wu MY, Liu YC, Tseng YH, Chang YS, Lin PJ, Wu TI. Pulmonary embolec tomy in high-risk acute pulmonary embolism: The effectiveness of a comprehensive therapeutic algorithm including extracorporeal life support. Resuscitation 2013; 84: 1365-70. doi: 10.1016/j.resuscitation.2013.03.032

22. Munakata R, Yamamoto $T$, Hosokawa $Y$, Tokita $Y$, Akutsu $K$, Sato N, et al. Massive pulmonary embolism requiring extracorporeal life support treated with catheter-based interventions. Int Heart J 2012; 53: 370-4. doi: 10.1536/ ihj.53.370 